\title{
Travel-time correction and preliminary results for ocean acoustic tomography in South China Sea
}

\author{
Xingyu $\mathrm{Ji}^{1}$, Hangfang Zhao ${ }^{1,2, *}$ \\ ${ }^{1}$ Department of Information Science and Electronic Engineering, Zhejiang University, 310027, Hangzhou, China \\ ${ }^{2}$ Key Laboratory of Ocean Observation-Imaging Testbed of Zhejiang Province, 316021, Zhoushan, China.
}

\begin{abstract}
An acoustic tomography trial experiment was conducted in South China Sea during May to August in 2016. Two moorings are installed apart from about $56.94 \mathrm{~km}$, while each consists of one low frequency source, 20 hydrophones deployed from the depth of about $400 \mathrm{~m}$ to $1600 \mathrm{~m}$, total 32 depth sensors and 3 compass and tilt sensors. Due to internal waves and currents in this area, as a typical value, horizontal drift of a mooring can reach $300 \mathrm{~m}$, thus moorings drift need to be considered to correct ray travel-time. In this paper, the shape of a mooring is estimated firstly and locations of all hydrophone array elements are then calculated and finally used to determine traveltime perturbation of acoustic arrivals. The mooring is modelled as 2 curves, while the end of the mooring is fixed at the cement anchor on the sea floor. Optimization is used to acquire hydrophone location inferential solution. The inferred shape of hydrophone array and element locations are used to correct the travel-times measured in the experiment. We find that corrected travel-times match the trend of the change of sound speed profile better in the sea. Finally, the corrected travel-times are used to tomography of sound speed profile. AR (Autoregressive) process is used to describe the dynamic evolution of sound speed profile and Kalman filter is applied in the sequential estimation. The performances of the time-independent method and the method using AR process and Kalman filter are compared, reasonably the latter is better than the former in particular with abundant measured data.
\end{abstract}

\section{Introduction}

Ocean acoustic tomography (OAT) allows to reconstruct the internal structure such as sound speed profile (SSP) of large ocean areas based on acoustic signals transmitting through these areas. Typical acoustic tomography is based on ray theory[1]. Ray travel times between source and receiver are used as measurements of acoustic propagation in inverse problem.

South China Sea is the largest adjacent sea in China. Generally speaking, it is surrounded by lots of land and connected with the external oceans through many straits between these lands. Thus, it is a typical local ocean system. The South China Sea is known as the natural internal wave test site[2]. We conducted an OAT experiment with the site position on the slope of the continental shelf where tides and current occur frequently during May to August in 2016. There are two moorings with one locates at $\mathrm{N} 21.32^{\circ} \mathrm{E} 119.406^{\circ}$ (marked as A1) and the other at $\mathrm{N} 20.846^{\circ} \mathrm{E} 119.2^{\circ}$ (marked as A2). The initial distance between them is $56.94 \mathrm{~km}$. The Diagram of the submerged buoy system is shown in Fig.1.

To measure ray travel times quite precisely to achieve about $10 \mathrm{~ms}$ time resolution, time keeping is a critical problem. Time error can be classified into time shift due to inaccurate clock and distance shift due to position change of source and receivers compared with reference position.

Array shape estimation and array element localization (AEL) is crucial for applications in OAT. Tomography experiments usually track the array elements by means of acoustic transponders deployed near the array[3]. However, the cost and size required for instrumenting a $1200-\mathrm{m}$ array with such sensors is prohibitive, hydrostatic pressure sensors for depth positioning along with few acoustic transponders and other compass and tilt sensors would be valuable in combination with an alternative method for subsequent positioning[4].

\section{Background and motivation}

Internal waves affect the OAT submerged buoy system. From data from 2016 experiment in South China Sea, more detail can be found in [5], the depth variety of the top TD in A1 submerged buoy can reach $60 \mathrm{~m}$ shown in Fig.2 and horizontal shift distance between long baseline system (LBL) and buoy centre can reach $300 \mathrm{~m}$ shown in Fig.3. As a typical example, one soliton internal wave is shown in Fig.4. Vertical depth of isotherm changes $76 \mathrm{~m}$ in 30 minutes which indicates that it is a concave internal solitary waves. As shown in Fig.5, the current velocity suddenly increases during the same time period. From the analysis of current velocity, there exist current with

* Corresponding author: hfzhao@zju.edu.cn 
period of about 28 days, one-day (23.54 hours) and halfday (12.64 hours) in Fig.6.

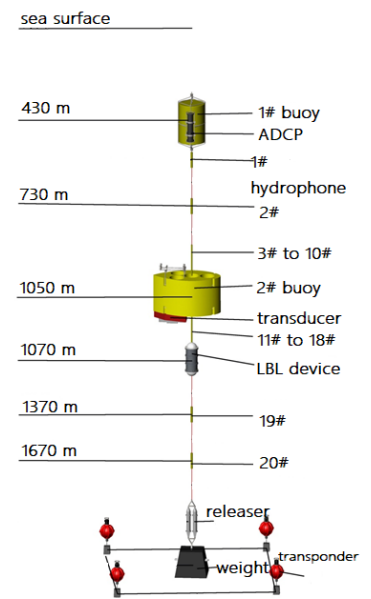

Fig. 1. Diagram of the submerged buoy system.

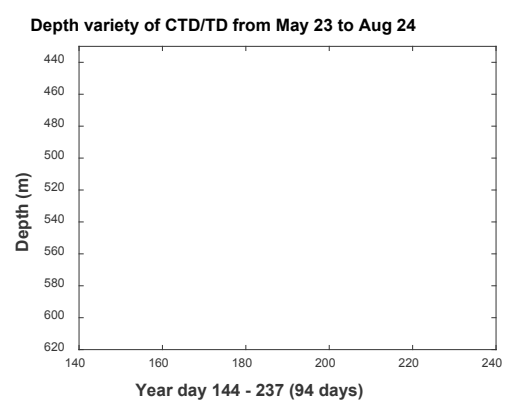

Fig. 2. Depth variety of the top TD in A1 mooring over experiment period.

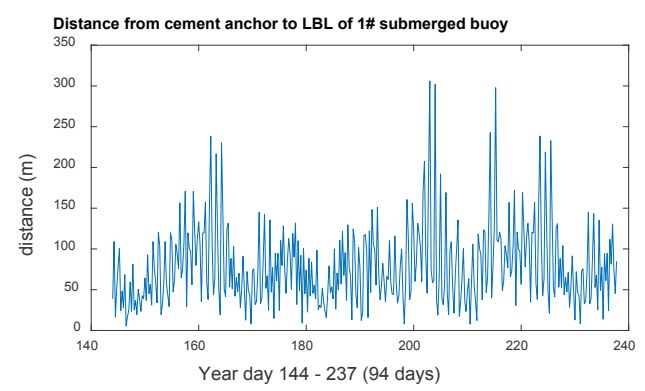

Fig. 3. Horizontal distance between cement anchor (mooring centre) to LBL system (near source) in A1 submerged buoy.

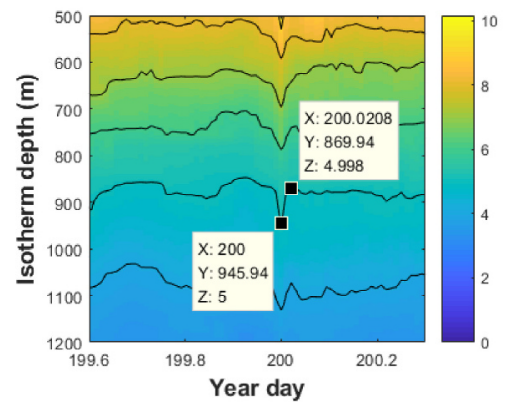

Fig. 4. One typical soliton internal wave with depth of $5{ }^{\circ} \mathrm{C}$ isotherm changes $76 \mathrm{~m}$ in 30 minutes.

For the problem of time shift, it is mainly solved by high precision clock in our OAT system. The time shift error of the selected high-precision clock is less than 3 ms per month. In 2016 experiment, the two clocks for 2 mooring systems are compared with GPS time on deck after three months' experiment and the errors were $2.7 \mathrm{~ms}$ and $9 \mathrm{~ms}$ respectively. Assuming that the time shift is linear during experiment period, we obtained time shift correction[6].

Positioning of the transmitting transducer (source) is carried out using LBL device with a beacon near it and 4 acoustic transponders on the sea bed. Positioning of receiving hydrophone array (receivers) uses array shape estimation algorithm with observed data including depths measured depth sensors, angles between mooring and vertical direction measured by compass and tilt sensors, GPS position of electronic cabin floating body and cement anchor measured by LBL.

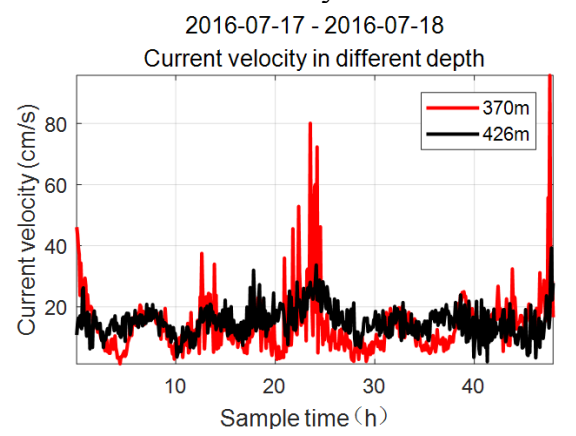

Fig. 5. Current velocity increase during the same time period as shown in Fig.4.

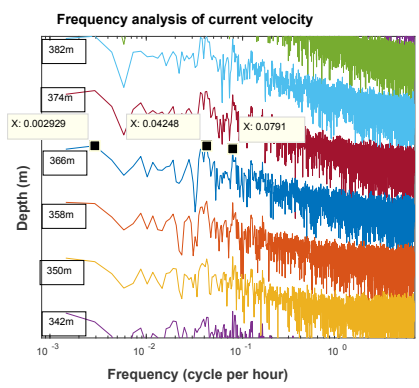

Fig. 6. Frequency analysis of current velocity. The peaks contain one-month, one-day and half-day (Marker points from left to right).

\section{Array shape estimation}

Each mooring contains 2 acoustic Doppler current profilers (ADCPs) locate at about $430 \mathrm{~m}$, one transmits upward and the other transmits downward. We use upward data only. Each mooring owns a LBL positioning system to locate the source and hydrophones. The mooring contains a buoy with ADCPs mounted at the top point, an electronic cabin floating body with acoustic source around the middle point, Kevlar rope and electronic cable and $1200 \mathrm{~kg}$ cement anchor on the seabed. The resultant force of buoyancy and gravity underwater is upward buoyancy which is $400 \mathrm{~kg}$ for the whole submerged buoy rope and cable. The electronic cabin floating body provides $150 \mathrm{~kg}$ buoyancy underwater. The buoyancy of ADCP buoy is smaller.

Fig. 7 shows the relationship of ocean mean current and depth in the area of OAT. Current is relatively small and constant deeper than the depth of $1000 \mathrm{~m}$ which is also near the sound channel axis and the position of source and electronic cabin floating body. On the other hand, the current is larger near the sea surface. So we 
model the submerged buoy as two parts, the upper half part and the lower half part. For the lower half part, the effect of current is ignored while gravity and upward buoyant force are the main considerations. For the upper half part, gravity and buoyant force are ignored while the force of current plays the leading role.

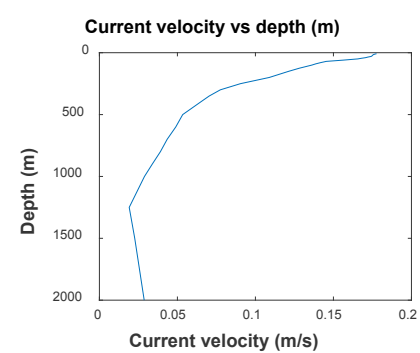

Fig. 7. Ocean mean current velocity and depth in the area of OAT (Data come from Argo and the figure shows an average value during a time period).

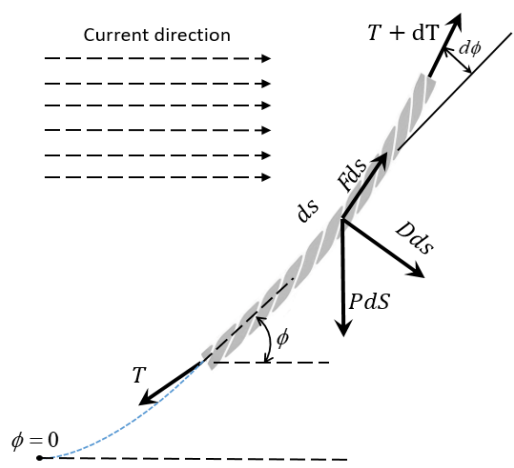

Fig. 8. Forces in cable element.

As shown in Fig.8, the forces on cable element with length $d s$ include tension $T$ and change of tension over $d s$, normal pressure drag $D d s$ on cable element, tangential friction drag $F d s$ on cable element (which can be ignored in the analysis), gravity force $P d s$ on cable element (including gravity force and buoyancy force), angle with flow direction $\phi$ and $d \phi$ is the change in angle $\phi$ over cable element length. Under static equilibrium, the resultant force of these forces must be zeros in all directions. In the normal direction

$$
(T+d T) \sin d \phi-D d s-P \cos \phi d s=0,
$$

given that $\phi$ is small enough, then $\sin d \phi \cong d \phi$, $d T d \phi \cong 0$

$$
T d \phi=(D+P \cos \phi) d s .
$$

In the tangential direction,

$$
-T+(T+d T) \cos d \phi-P \sin \phi d s=0,
$$

and similarly, we have

$$
d T=P \sin \phi d s
$$

If the gravity force $P$ is predominant and normal pressure drag $D$ can be neglected, as well as $P \gg D$, Eq.(2) and Eq.(4) can be reduced to

$$
\begin{gathered}
T d \phi=P \cos \phi d s, \\
d T=P \sin \phi d s,
\end{gathered}
$$

Combining Eq.(5) and Eq.(6), integrating of $\phi$ from $\phi=0$ and using geometric relation, we can derive that

$$
\begin{gathered}
y=\int d y=\frac{T_{0}}{P}\left[\cosh \left(\frac{P x}{T_{0}}\right)-1\right], \\
s=\frac{T_{0}}{P} \sinh \left(\frac{P x}{T_{0}}\right),
\end{gathered}
$$

where $T_{0}$ is the tension at the origin $\phi=0, P$ is the chain weight per length.

Similarly, if normal pressure drag $D$ is predominant and the gravity force $P$ can be neglected, as well as $P \ll D$,

$$
\begin{gathered}
T d \phi=D d s, \\
\frac{d T}{d s}=0,
\end{gathered}
$$

Define $R=D / \sin ^{2} \phi$, we can get the results

$$
\begin{gathered}
s=\frac{T_{0}}{R}\left(\cot \phi_{1}-\cot \phi_{2}\right), \\
x=-\frac{T_{0}}{R}\left(\operatorname{cosec} \phi_{2}-\operatorname{cosec} \phi_{1}\right), \\
y=\frac{T_{0}}{R}\left[\ln \left(\tan \frac{\phi_{2}}{2}\right)-\ln \left(\tan \frac{\phi_{1}}{2}\right)\right],
\end{gathered}
$$

where $T_{0}$ is tension at any point of the cable considering that tension $T$ is constant over the whole cable from Eq.(9), $\phi_{1}$ and $\phi_{2}$ are angle with flow direction of start point and end point on the cable, $s$ is cable length ${ }^{[7]}$.

We optimize parameters by minimize

$$
\min _{\phi, T_{0}, R \text { or } P} s-\hat{s}
$$

where $\hat{s}$ is obtained by given a set of parameters in interested domain region.

\section{Experiment results}

\subsection{Array shape estimation}

We search parameter of $\phi$ in the region around vertical angle measured by compass and tilt sensors. For one time index of day 216, the estimated shape of the array is shown in Fig.9. Current is main effect for the upper half part while buoyant force for the lower half. So the two parts have different bending directions. We also apply statistical method to evaluate estimation performance over time (average over all hydrophones in various depth) and over depth (average over all hydrophones in various depth). From Fig.10 and Fig.11, RMSE is less than $2 \mathrm{~m}$ in a majority of time and decrease with depth increasing on the whole which is also reasonable because the reference point is chosen on the seabed. RMSE (Root Mean Square Error) is defined as 


$$
R M S E=\sqrt{\frac{1}{M} \sum_{i=1}^{M}\left(\hat{y}_{i}-y_{i}\right)^{2}}
$$

where $M$ is the number of data, $y_{i}$ is the depth of each pressure sensor and $\hat{y}_{i}$ is estimated value by array shape estimation algorithm.

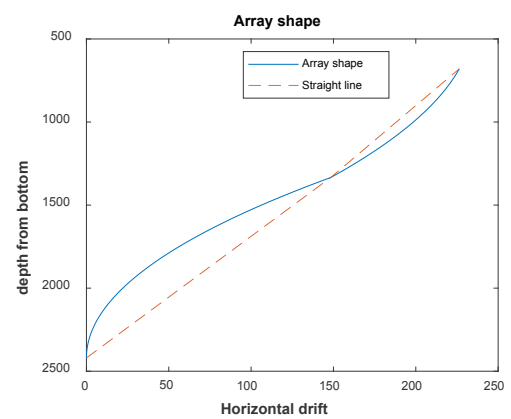

Fig. 9. Estimated mooring array shape in 216-th day.

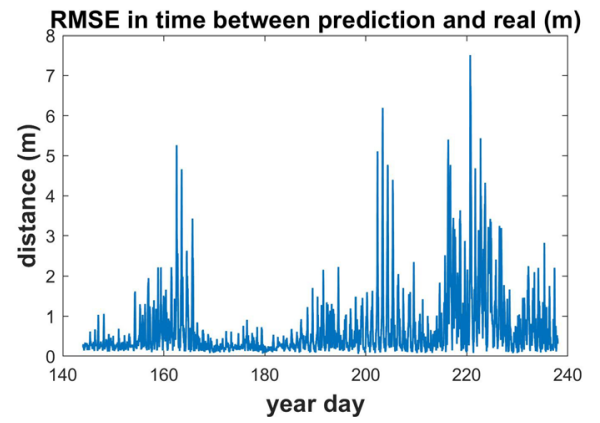

Fig. 10. RMSE between estimated depth and measured depth, averaged over all hydrophones.

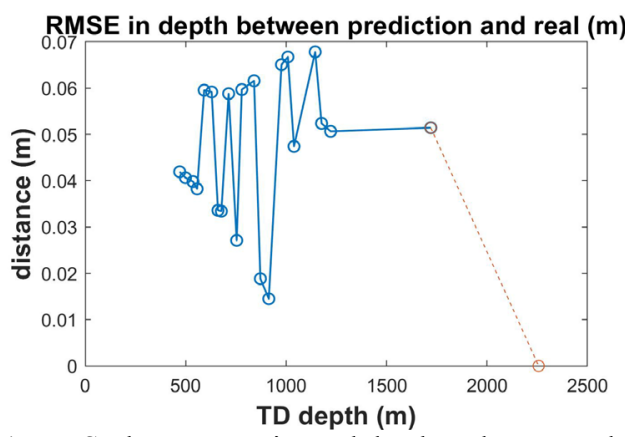

Fig. 11. RMSE between estimated depth and measured depth, averaged over all time index.

In order to localize the position of the array in $3 \mathrm{D}$ sea, the direction of cement anchor and LBL is calculated and we model the array on this vertical plane.

\subsection{Travel-time correction}

With the array shape in analytical expression, we can calculate horizontal drift of each hydrophone.

We calculate the difference value of time-delay between reference and estimated environment setting including source and receiver depth and range. Raw travel-times are corrected by minus difference value resulting in corrected travel-times. In Fig.12, the dots are delay-time peaks of rays with dot size proportional to SNR in $1008.5 \mathrm{~m}$ near source and acoustic axis channel. Before 210-th day, corrected travel-times are smoother and aggregated, especially stable for the significant travel-times increase between 180-190 days which corresponds to significant depth variety in Fig. 2.
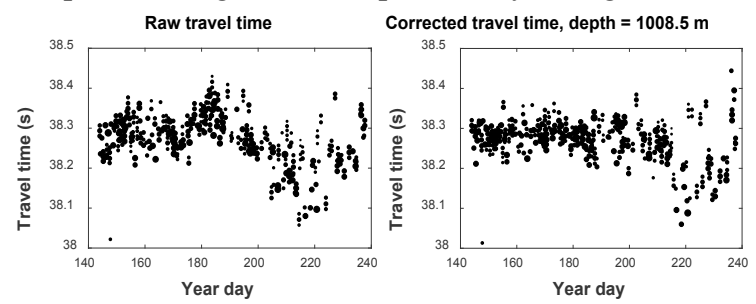

Fig. 12. Raw and corrected ray travel-times in $1008.5 \mathrm{~m}$ near source. The dots are delay-time peaks of rays with dot size proportional to SNR.

\subsection{OAT inverse results}

SSP in fixed point can be expressed as the sum of basic functions. We choose EOF (Empirical Orthogonal Function) as basis functions, so

$$
c_{l}(\mathbf{r})=\bar{c}(\mathbf{r})+\sum_{k=1}^{K} \alpha_{l, k} \mathbf{f}_{k}(\mathbf{r}), \quad 1 \leq l \leq L
$$

where $l$ is time index and $L$ is number of SSP in different time index, $K$ is the number of basis functions, $\alpha_{l, k}$ is coefficient of basis functions in time index $l$, $\bar{c}(\mathbf{r})$ is mean SSP over $c_{l}(\mathbf{r}), \mathbf{f}_{k}(\mathbf{r})$ is $k$-th basis function.

We model the evolution of sound speed profile as AR process which is equivalent to Gauss-Markov process, which can be expressed as

$$
c_{l+1}(\mathbf{r})=\mathbf{a}_{l} c_{l}(\mathbf{r})+n_{l}
$$

thus coefficients of basis functions are

$$
\mathbf{x}_{l+1}=\mathbf{a}_{1} \mathbf{x}_{l}+\mathbf{n}_{l}
$$

where $\mathbf{a}_{l}=\operatorname{diag}\left(a_{l, 1}, a_{l, 2}, \ldots, a_{l, K}\right), \mathbf{n}_{l}$ is noise and $\mathbf{x}_{l}=\left[\alpha_{l, 1}, \alpha_{l, 2}, \cdots, \alpha_{l, K}\right]^{T}$. Coefficients can be calculated by Burg algorithm with historical data.

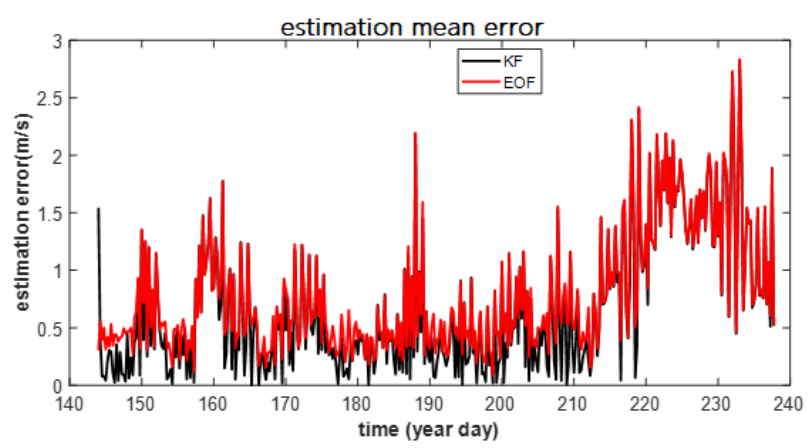

Fig. 13. Mean error of estimated and measured SSP, averaged over depth. KF is Kalman filter method.

We choose 1-order AR model and 6-order EOF and applying Kalman filter (KF) with corrected travel-times for inverse procedure. We compare EOF method which calculates each time index independently and KF method which calculates sequentially. Results are shown in Fig.13, where mean error is the average error over depth between measured SSP by TD and CTD and estimated SSP. Between 143-210 day, the mean error of KF 
method is less than EOF method because KF method can make use the evolution information of SSP to perform better. After that time period, both of the methods have poor performance because lack of delay-time data suffering from too low SNR to detect peak.

\section{Conclusions}

OAT in South China Sea is faced with a series of challenges, internal wave and current affect the submerged buoy system resulting that the array shape and element position change a lot. Some examples are shown to confirm the existence and effect. Considering the current velocity is this area, an array shape estimation method is proposed using one transponder system, 3 compass and tilt sensors and many depth sensors. The performance of this method is evaluated to be acceptable. Corrected ray travel-times are applied in inverse procedure, and the performance of Kalman filter combined with AR process is relatively better in sequential SSP tomography in a time period.

The author would like to thank Hangzhou Applied Acoustic Research Institute for sharing the OAT data analysed in this paper. This work was supported by the National Key R\&D Program of China (Grant No.2016YFC1400100)

\section{References}

1. M. Walter, P. Worcester and C. Wunsch, Ocean acoustic tomogragphy (Cambridge university press, 1995)

2. Z. M. Li, S.Q. Cai, J. Chen, R.Y. Chen, D. X. Wang, and Y. Du, J. Trop. Oceanogr., 33, 1 (2014): 10-16.

3. T. K. Chandrayadula, and E. W. Kathleen MTS/IEEE Oceans 2008 conference, (Quebec, Canada. 2008)

4. B. J. Sotirin, and J. A. Hildebrand. J. Acoust. Soc. Am. 87, 1 (1990): 154-167.

5. H.F. Zhao, X.Y. Ji, Y. Qiu, and Y. Huang, 4th Underwater Acoustics Conference and Exhibition UACE2017, (Skiathos, Greece, 2017)

6. X. W. Ying, T.J. Xia, and G. J. Jiang 4th Underwater Acoustics Conference and Exhibition - UACE2017, (Skiathos, Greece, 2017)

7. H. O Berteaux, Buoy engineering. (Wiley, New York, 1976) 